\title{
Synthesis and antitumor activity of novel pyrazolo[1,5-a]pyrimidine derivatives
}

\author{
Mervat Mostafa El-Enanya, Mona Monir Kamelb, \\ Omneya Mahmoud Khalilb and Hala Bakr El-Nassan',* \\ a Pharmacology Chemistry Department, Faculty of Pharmaceutical Science and Pharmaceutical Industries, Future University, Cairo, 11562, Egypt** \\ b Organic Chemistry Department, Faculty of Pharmacy, Cairo University, Cairo, 11562, Egypt \\ *Corresponding author at: Organic Chemistry Department, Faculty of Pharmacy, Cairo University, Cairo, 11562, Egypt. Tel.: +00202.23632245; \\ fax: +00202.23635140.E-mail address: hala_bakr@hotmail.com (H.B.El-Nassan). \\ ** Sabbatical leave from Cairo University.
}

\section{ARTICLE INFORMATION}

Received: 14 November 2010

Received in revised form: 21 February 2011

Accepted: 30 March 2011

Online: 30 September 2011

\section{KEYWORDS}

Pyrazole

5-Aminopyrazole

Pyrazolo[1,5- $a]$ pyrimidine

Antitumor activity

Cytotoxic activity

HCT116

\section{Introduction}

Cancer is defined as malignant growth of cells. Most tumors arise from a combination of genetic mutations in the cell. These genetic changes lead to activation of oncogenes and suppression or deletion of tumor suppressor genes. As a result, there is unregulated cell proliferation and also a delay in programmed cell death, apoptosis [1]. Most of the clinically used antineoplastic drugs aim to suppress the proliferative process (e.g.: DNA replication or chromosome segregation) [1].

Pyrazolo[1,5-a]pyrimidines are of considerable chemical and pharmacological importance as purine analogs and many derivatives of pyrazolo[1,5- $a$ ]pyrimidines have been reported to exhibit cytotoxic activity [2-10]. Different mechanisms account for the cytotoxic effect of this class of compounds, where they have been reported to act as vascular endothelial growth factor receptor inhibitor [2] and cyclin dependent kinase inhibitors [3,6-8].

Several 7-substituted aminopyrazolo[1,5-a]pyrimidine derivatives were reported to have antiproliferative activity against HCT116 and other cell lines (e.g. compounds 1-3) [3,6,8] (Figure 1).

In the present study, several pyrazolo[1,5- $a$ ]pyrimidine derivatives, bearing 2-methylsulphanyl group, 3-nitrile group and 7-amino (6a, 6b, 7a-d, 8 and 9) or 7-substituted amino group (14ac) were prepared. Besides, different substitutions were introduced at position 5 (aromatic ring, amino group or carbonyl group) (Scheme 1 and 2).

Meanwhile, the study aimed to synthesize 7-substituted amino5-methylpyrazolo[1,5-a]pyrimidine derivatives. Nevertheless, the product obtained using two different reaction conditions was the 7methyl derivative.

\section{Experimental}

\subsection{Instrumentation}

Melting points were determined using a Griffin apparatus and were uncorrected. IR spectra were recorded on Mattson Genesis II FT-IR and values were represented in $\mathrm{cm}^{-1} .{ }^{1} \mathrm{H}$ NMR were carried out on Varian Gemini $200 \mathrm{MHz}$ spectrophotometer, Microanalytical center, Cairo University, Cairo, Egypt, using TMS as an internal standard and chemical shifts were recorded in ppm on $\delta$ scale and coupling constants $($ ) are given in Hz. The electron impact (EI) mass spectra were recorded on Shimadzu QP-2010 plus, Microanalytical center, Cairo University, Cairo, Egypt. Analytical thin layer chromatography (TLC) on silica gel plates containing UV indicator was employed routinely to follow the course of reactions and to check the purity of products. All reagents and solvents were purified and dried by standard techniques. Elemental microanalyses were performed at Microanalytical Center, Cairo University, Cairo, Egypt, and were within $\pm 0.4 \%$.

\subsection{Synthesis}

2.2.1. General procedure for the synthesis of 7-amino-2-methyl sulphanyl-5-(substituted phenyl)-4,5-dihydropyrazolo [1,5-a] pyrimidine-3,6-dicarbonitriles $6 a, b$ and 7-amino-2-methyl sulphanyl-5-(substituted phenyl)pyrazolo[1,5-a]pyrimidine-3,6dicarbonitriles $7 a-d$

A mixture of 5-amino-3-methylsulphanyl-1H-pyrazole-4carbonitrile (4) [11] $(0.31 \mathrm{~g}, 0.002 \mathrm{~mol})$, the appropriate substituted benzylidenemalononitrile 5a-f [12-15] (0.002 mol) and triethylamine $(2 \mathrm{~mL})$ in absolute ethanol $(20 \mathrm{~mL})$ was heated under reflux for $7 \mathrm{~h}$. The precipitate formed was filtered, dried and crystallized from acetic acid.

7-Amino-5-(2-chlorophenyl)-2-methylsulphanyl-4,5-dihydro pyrazolo[1,5-a]pyrimidine-3,6-dicarbonitrile (6a): Yield: 33\%. M.p.: 246-247 ${ }^{\circ} \mathrm{C}$. FT-IR $\left(\mathrm{cm}^{-1}\right): 3453,3307,3236\left(\mathrm{NH} / \mathrm{NH}_{2}\right), 2228,2192$ (CN). ${ }^{1} \mathrm{H}$ NMR $\left(200 \mathrm{MHz}, \mathrm{DMSO}-d_{6}, \delta \mathrm{ppm}\right): 2.6\left(\mathrm{~s}, 3 \mathrm{H}, \mathrm{SCH}_{3}\right), 5.7$ (d, 

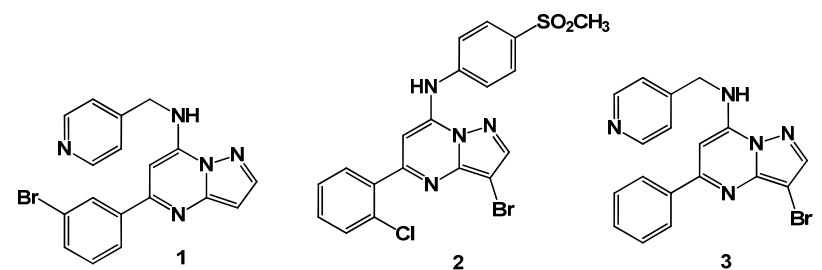

Figure 1. Examples of 7-substituted aminopyrazolo[1,5-a]pyrimidines with antiproliferative activity.

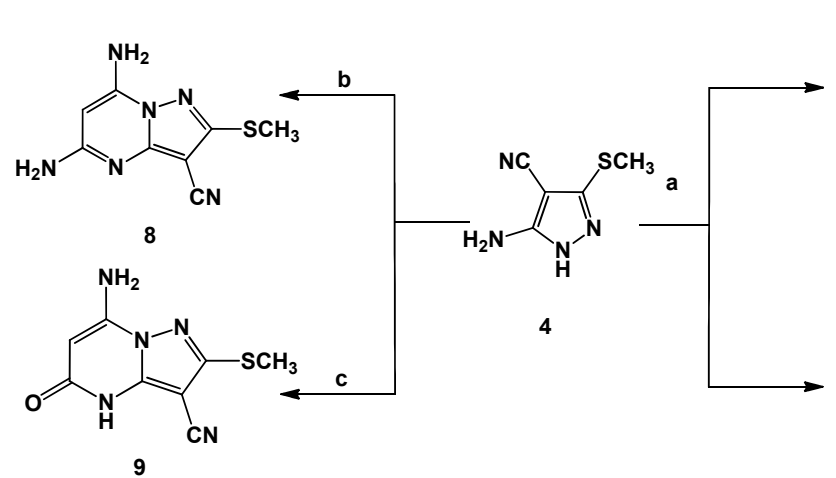<smiles>CSc1nn2c(c1C#N)NC(C)C(C#N)=C2N</smiles>

$6 a, b$<smiles>CSc1nn2c(N)c(C#N)c(Br)nc2c1C#N</smiles>

7a-d

\begin{tabular}{c|ccc|c} 
Compound & $\mathrm{Ar}$ & & Compound & $\mathrm{Ar}$ \\
, $6 a$ & $2-\mathrm{ClC}_{6} \mathrm{H}_{4}$ & & $5 d, 7 b$ & $3-\mathrm{NO}_{2} \mathrm{C}_{6} \mathrm{H}_{4}$ \\
$5 b, 6 b$ & $2-\mathrm{CH}_{3} \mathrm{OC}_{6} \mathrm{H}_{4}$ & & $5 e, 7 c$ & $4-\mathrm{ClC}_{6} \mathrm{H}_{4}$ \\
$5 c, 7 a$ & $2-\mathrm{OHC}_{6} \mathrm{H}_{4}$ & & $5 f, 7 d$ & $4-\left(\mathrm{CH}_{3}\right)_{2} \mathrm{NC}_{6} \mathrm{H}_{4}$
\end{tabular}

Reagents: a) $\mathrm{ArCH}=\mathrm{CH}(\mathrm{CN})_{2}$ 5a-f, triethylamine, ethanol; b) $\mathrm{CH}_{2}(\mathrm{CN})_{2}$, triethylamine, ethanol; c) $\mathrm{NCCH}_{2} \mathrm{COOC}_{2} \mathrm{H}_{5}$, Fusion at $160^{\circ} \mathrm{C}$.

Scheme 1

$1 \mathrm{H}, H 5, J=2 \mathrm{~Hz}), 7.3\left(\mathrm{~s}, 1 \mathrm{H}, \mathrm{N} H, \mathrm{D}_{2} \mathrm{O}\right.$ exchangeable), 7.4-7.5 (m, 4H, Ar- $H$ ), $9.2\left(\mathrm{~s}, 2 \mathrm{H}, \mathrm{NH}_{2}, \mathrm{D}_{2} \mathrm{O}\right.$ exchangeable). Anal. Calcd. for $\mathrm{C}_{15} \mathrm{H}_{11} \mathrm{ClN}_{6} \mathrm{~S}$ : C, 52.55; H, 3.23; N, 24.51. Found: C, 52.62; H, 3.44; N, $24.27 \%$.

7-Amino-5-(2-methoxyphenyl)-2-methylsulphanyl-4,5-dihydro pyrazolo[1,5-a]pyrimidine-3,6-dicarbonitrile (6b): Yield: 48\%. M.p.: 244-245 ${ }^{\circ} \mathrm{C}$. FT-IR $\left(\mathrm{cm}^{-1}\right): 3425,3277,3219\left(\mathrm{NH} / \mathrm{NH}_{2}\right), 2218,2189$ $(\mathrm{CN}) .{ }^{1} \mathrm{H}$ NMR $\left(200 \mathrm{MHz}, \mathrm{DMSO}-d_{6}, \delta \mathrm{ppm}\right): 2.6\left(\mathrm{~s}, 3 \mathrm{H}, \mathrm{SCH} \mathrm{H}_{3}\right), 3.7(\mathrm{~s}$ $\left.3 \mathrm{H}, \mathrm{OCH}_{3}\right), 5.4(\mathrm{~s}, 1 \mathrm{H}, H 5), 7.2\left(\mathrm{~s}, 1 \mathrm{H}, \mathrm{N} H, \mathrm{D}_{2} \mathrm{O}\right.$ exchangeable), 6.9-7.3 (m, 4H, Ar- $H$ ), 9.0 (s, 2H, N $H_{2}, \mathrm{D}_{2} \mathrm{O}$ exchangeable). MS (m/z (\%)): 338 [M+, 1.12\%]. Anal. Calcd. for $\mathrm{C}_{16} \mathrm{H}_{14} \mathrm{~N}_{6} \mathrm{OS}$ : C, 56.79; $\mathrm{H}, 4.17$; N, 24.83. Found: C, 56.50; H, 3.84; N, 24.97\%.

7-Amino-5-(2-hydroxyphenyl)-2-methylsulphanylpyrazolo[1,5-a] pyrimidine-3,6-dicarbonitrile (7a): Yield: $22 \%$. M.p.: $224-225^{\circ} \mathrm{C}$. FTIR $\left(\mathrm{cm}^{-1}\right): 3436,3344\left(\mathrm{NH}_{2}\right), 2200(\mathrm{CN}) .{ }^{1} \mathrm{H}$ NMR $(200 \mathrm{MHz}, \mathrm{DMSO}-$ $\left.d_{6}, \delta \mathrm{ppm}\right): 2.7\left(\mathrm{~s}, 3 \mathrm{H}, \mathrm{SC} H_{3}\right), 7.1-8.2(\mathrm{~m}, 4 \mathrm{H}, \mathrm{Ar}-H), 8.9\left(\mathrm{~s}, 2 \mathrm{H}, \mathrm{N} H_{2}\right.$, $\mathrm{D}_{2} \mathrm{O}$ exchangeable), $9.3\left(\mathrm{~s}, 1 \mathrm{H}, \mathrm{OH}, \mathrm{D}_{2} \mathrm{O}\right.$ exchangeable). Anal. Calcd. for $\mathrm{C}_{15} \mathrm{H}_{10} \mathrm{~N}_{6} \mathrm{OS}$ : C, 55.89; H, 3.12; N, 26.07. Found: C, 56.02; H, 3.59; $\mathrm{N}, 26.23 \%$.

7-Amino-2-methylsulphanyl-5-(3-nitrophenyl)pyrazolo[1,5-a] pyrimidine-3,6-dicarbonitrile (7b): Yield: $44 \%$. M.p.: $287-288^{\circ} \mathrm{C}$. FTIR ( $\left.\mathrm{cm}^{-1}\right): 3308,3273\left(\mathrm{NH}_{2}\right), 2214,2131(\mathrm{CN}), 1528,1345\left(\mathrm{NO}_{2}\right) .{ }^{1} \mathrm{H}$ NMR (200 MHz, DMSO- $d_{6}, \delta$ ppm): $2.7\left(\mathrm{~s}, 3 \mathrm{H}, \mathrm{SCH} H_{3}\right), 7.8(\mathrm{t}, 1 \mathrm{H}, J=8$ Hz, Ar- $H$ ), 8.3 (d, 1H, J=7.8 Hz, Ar- $H$ ), 8.4 (d, 1H, $J=8$ Hz, Ar- $H$ ), 8.6 (s, $1 \mathrm{H}, \mathrm{Ar}-\mathrm{H}$ ), 9.4 (br s, $2 \mathrm{H}, \mathrm{NH}_{2}, \mathrm{D}_{2} \mathrm{O}$ exchangeable). Anal. Calcd. for $\mathrm{C}_{15} \mathrm{H}_{9} \mathrm{~N}_{7} \mathrm{O}_{2} \mathrm{~S}$ : C, 51.27; H, 2.58; N, 27.90. Found: C, 51.71; H, 2.30; N, $27.80 \%$.
7-Amino-5-(4-chlorophenyl)-2-methylsulphanylpyrazolo[1,5-a] pyrimidine-3,6-dicarbonitrile (7c): Yield: 45\%. M.p.: 273-274 ${ }^{\circ} \mathrm{C}$. FTIR ( $\left.\mathrm{cm}^{-1}\right): 3439,3297\left(\mathrm{NH}_{2}\right), 2222(\mathrm{CN}) .{ }^{1} \mathrm{H}$ NMR $(200 \mathrm{MHz}$, DMSO$\left.d_{6}, \delta \mathrm{ppm}\right): 2.7\left(\mathrm{~s}, 3 \mathrm{H}, \mathrm{SC} H_{3}\right), 7.6(\mathrm{~d}, 2 \mathrm{H}, J=7.6 \mathrm{~Hz}, \operatorname{Ar}-H), 7.8(\mathrm{~d}, 2 \mathrm{H}$ $J=7.6 \mathrm{~Hz}$, Ar- $H$ ), 9.3 (br s, $2 \mathrm{H}, \mathrm{NH}_{2}, \mathrm{D}_{2} \mathrm{O}$ exchangeable). MS (m/z (\%)): $342\left[(\mathrm{M}+2)^{+}, 38.96 \%\right], 340\left[\mathrm{M}^{+}, 100 \%\right]$. Anal. Calcd. for $\mathrm{C}_{15} \mathrm{H}_{9} \mathrm{ClN}_{6} \mathrm{~S}$ : C, 52.86; H, 2.66; N, 24.65. Found: C, 52.70; H, 2.30; $\mathrm{N}$ 24.86.

7-Amino-5-(4-dimethylaminophenyl)-2-methylsulphanylpyrazolo [1,5-a]pyrimidine-3,6-dicarbonitrile (7d): Yield: 32\%. M.p.: 280-281 ${ }^{\circ} \mathrm{C}$. FT-IR $\left(\mathrm{cm}^{-1}\right)$ : 3449, $3370\left(\mathrm{NH}_{2}\right), 2209(\mathrm{CN}) .{ }^{1} \mathrm{H}$ NMR $(200 \mathrm{MHz}$ DMSO- $d_{6}, \delta$ ppm): $2.7\left(\mathrm{~s}, 3 \mathrm{H}, \mathrm{SCH}_{3}\right), 3.0\left(\mathrm{~s}, 6 \mathrm{H},-\mathrm{N}\left(\mathrm{CH}_{3}\right)_{2}\right), 6.7(\mathrm{~d}, 2 \mathrm{H}$ $J=8.8 \mathrm{~Hz}, \operatorname{Ar}-H$ ), 7.8 (d, 2H, J=9.2 Hz, Ar- $H$ ), 9.0 (br s, $2 \mathrm{H}, \mathrm{NH}_{2}, \mathrm{D}_{2} \mathrm{O}$ exchangeable). Anal. Calcd. for $\mathrm{C}_{17} \mathrm{H}_{15} \mathrm{~N}_{7} \mathrm{~S}$ : C, 58.43; $\mathrm{H}, 4.32 ; \mathrm{N}$, 28.05. Found: C, 58.73; H, 4.64; N, 28.30\%

\subsubsection{5,7-Diamino-2-methylsulphanylpyrazolo[1,5-a]} pyrimidine-3-carbonitrile (8)

A mixture of 5-amino-3-methylsulphanyl-1H-pyrazole-4carbonitrile (4) (0.77 g, $0.005 \mathrm{~mol})$, malononitrile $(0.33 \mathrm{~g}, 0.005$ mol) and triethylamine $(2 \mathrm{~mL})$ in absolute ethanol $(30 \mathrm{~mL})$ was heated under reflux for $10 \mathrm{~h}$. The precipitate formed was filtered, dried and crystallized from acetic acid. Yield: 26\%. M.p.: 254-255 ${ }^{\circ} \mathrm{C}$. FT-IR (cm-1): 3479, 3431, 3373, $3317\left(\mathrm{NH}_{2}\right), 2203(\mathrm{CN}) .{ }^{1} \mathrm{H}$ NMR (200 MHz, DMSO- $d_{6}, \delta \mathrm{ppm}$ ): 2.6 (s, 3H, $\mathrm{SCH}_{3}$ ), 5.3 (s, 1H, H6), 6.7 (s, $2 \mathrm{H}, \mathrm{NH}_{2}, \mathrm{D}_{2} \mathrm{O}$ exchangeable), $7.3\left(\mathrm{~s}, 2 \mathrm{H}, \mathrm{NH}_{2}, \mathrm{D}_{2} \mathrm{O}\right.$ exchangeable). Anal. Calcd. for $\mathrm{C}_{8} \mathrm{H}_{8} \mathrm{~N}_{6} \mathrm{~S}$ : C, 43.62; $\mathrm{H}, 3.66$; N, 38.15. Found: $\mathrm{C}, 43.99$; H, 3.55; N, 38.60\% 


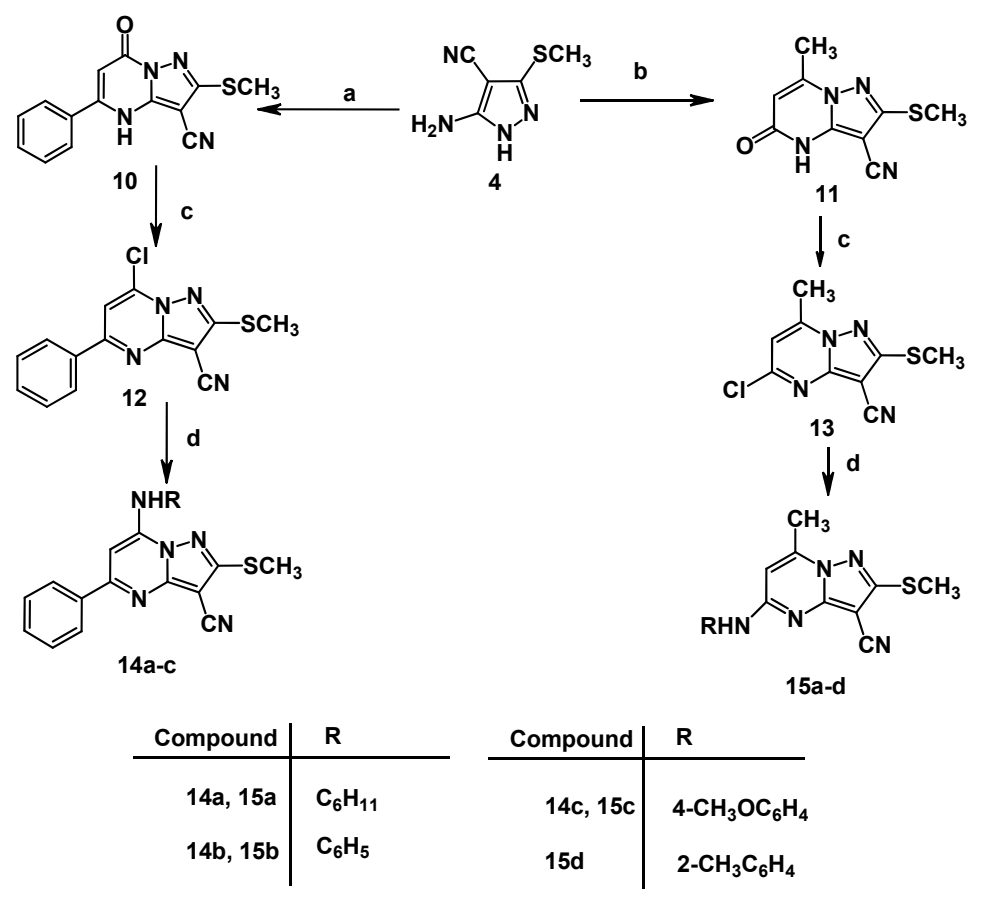

Reagents: a) $\mathrm{C}_{6} \mathrm{H}_{5} \mathrm{COCH}_{2} \mathrm{COOC}_{2} \mathrm{H}_{5}$, Fusion at $160^{\circ} \mathrm{C}$;

b) $\mathrm{CH}_{3} \mathrm{COCH}_{2} \mathrm{COOC}_{2} \mathrm{H}_{5}$, Fusion at $170^{\circ} \mathrm{C}$ (method A), acetic acid (method B);

c) $\mathrm{POCl}_{3}$;

d) $\mathrm{RNH}_{2}$, triethylamine, ethanol.

Scheme 2

2.2.3. 7-Amino-2-methylsulphanyl-5-oxo-4,5-dihydropyrazolo [1,5-a]pyrimidine-3-carbonitrile (9)

A mixture of 5-amino-3-methylsulphanyl-1 $H$-pyrazole-4carbonitrile (4) (2.31 g, $0.015 \mathrm{~mol}$ ) and ethyl cyanoacetate (1.69 g, $1.70 \mathrm{~mL}, 0.015 \mathrm{~mol}$ ) was heated at $160{ }^{\circ} \mathrm{C}$ in an oil bath for $2 \mathrm{~h}$. The solid formed was triturated with ethanol $(10 \mathrm{~mL})$, filtered, dried and crystallized from DMF. Yield: $93 \%$. M.p.: $>300^{\circ} \mathrm{C}$. FT-IR $\left(\mathrm{cm}^{-1}\right)$ : 3433, 3319 (NH/NH2), 2220 (CN), 1650 (CO). ${ }^{1} \mathrm{H}$ NMR (200 MHz, DMSO- $d_{6}, \delta$ ppm): $2.6\left(\mathrm{~s}, 3 \mathrm{H}, \mathrm{SCH}_{3}\right), 5.2(\mathrm{~d}, 1 \mathrm{H}, H 6, J=2.2 \mathrm{~Hz}), 7.6(\mathrm{~s}$, $2 \mathrm{H}, \mathrm{NH}_{2}, \mathrm{D}_{2} \mathrm{O}$ exchangeable), 11.9 (br s, $1 \mathrm{H}, \mathrm{N} H, \mathrm{D}_{2} \mathrm{O}$ exchangeable). Anal. Calcd. for $\mathrm{C}_{8} \mathrm{H}_{7} \mathrm{~N}_{5} \mathrm{OS}$ : C, 43.43; H, 3.18; N, 31.65. Found: C, 43.01; H, 3.36; N, 31.22\%.

\subsubsection{2-Methylsulphanyl-5-phenyl-7-oxo-4,7-dihydropyrazolo [1,5-a]pyrimidine-3-carbonitrile (10)}

A mixture of 5-amino-3-methylsulphanyl- $1 H$-pyrazole-4carbonitrile (4) (2.31 g, $0.015 \mathrm{~mol})$ and ethyl benzoylacetate (2.88 g, $2.60 \mathrm{~mL}, 0.015 \mathrm{~mol}$ ) was heated at $160^{\circ} \mathrm{C}$ in an oil bath for $2 \mathrm{~h}$. The solid formed was triturated with ethanol $(10 \mathrm{~mL})$, filtered, dried and crystallized from acetic acid. Yield: 67\%. M.p.: 268-269 ${ }^{\circ} \mathrm{C}$. FT-IR ( $\mathrm{cm}^{-1}$ ): 3466 (NH), 2222 (CN), 1718 (CO). ${ }^{1} \mathrm{H}$ NMR (200 $\left.\mathrm{MHz}, \mathrm{DMSO}-d_{6}, \delta \mathrm{ppm}\right): 2.6\left(\mathrm{~s}, 3 \mathrm{H}, \mathrm{SC} H_{3}\right), 6.2\left(\mathrm{~s}, 1 \mathrm{H}, \mathrm{N} H, \mathrm{D}_{2} \mathrm{O}\right.$ exchangeable), 7.5-7.8 (m, 6H, Ar-H+ H6). Anal. Calcd. for $\mathrm{C}_{14} \mathrm{H}_{10} \mathrm{~N}_{4} \mathrm{OS}$ : C, 59.56; H, 3.57; N, 19.84. Found: C, 59.97; H, 3.57; N, $20.16 \%$.

\subsubsection{7-Methyl-2-methylsulphanyl-5-oxo-4,5-dihydropyrazolo} [1,5-a]pyrimidine-3-carbonitrile (11)

Method A: A mixture of 5-amino-3-methylsulphanyl-1Hpyrazole-4-carbonitrile (4) (2.31 g, $0.015 \mathrm{~mol}$ ) and ethyl acetoacetate $(1.95 \mathrm{~g}, 1.90 \mathrm{~mL}, 0.015 \mathrm{~mol})$ was heated at $170{ }^{\circ} \mathrm{C}$ in an oil bath for $2 \mathrm{~h}$. The solid product was triturated with ethanol $(10 \mathrm{~mL})$, filtered, dried and crystallized from acetic acid.

Method B: A mixture of 5-amino-3-methylsulphanyl- $1 \mathrm{H}$ pyrazole-4-carbonitrile (4) (2.31 g, $0.015 \mathrm{~mol})$ and ethyl acetoacetate $(1.95 \mathrm{~g}, 1.90 \mathrm{~mL}, 0.015 \mathrm{~mol}$ ) in glacial acetic acid (50 $\mathrm{mL}$ ) was heated under reflux for $15 \mathrm{~h}$. The solid formed was filtered, dried and crystallized from acetic acid.

Yield: 90\% (Method A), 77\% (Method B). M.p.: 300-301 ${ }^{\circ} \mathrm{C}$ [16]. FT-IR (cm-1): $3470(\mathrm{NH}), 2225(\mathrm{CN}), 1661$ (CO). ${ }^{1} \mathrm{H}$ NMR (200 MHz, DMSO- $\left.d_{6}, \delta \mathrm{ppm}\right): 2.2\left(\mathrm{~s}, 3 \mathrm{H}, \mathrm{CH}_{3}\right), 2.6\left(\mathrm{~s}, 3 \mathrm{H}, \mathrm{SCH}_{3}\right), 5.8(\mathrm{~s}, 1 \mathrm{H}, H 6)$, $13.2\left(\mathrm{~s}, 1 \mathrm{H}, \mathrm{N} H, \mathrm{D}_{2} \mathrm{O}\right.$ exchangeable).

\subsubsection{General procedure for the synthesis of pyrazolo[1,5-a] pyrimidines 12 and 13}

A mixture of pyrazolo[1,5- $a$ ]pyrimidine derivatives $\mathbf{1 0}$ or $\mathbf{1 1}$ $(0.004 \mathrm{~mol})$ and phosphorus oxychloride $(20 \mathrm{~mL})$ was heated under reflux for $7 \mathrm{~h}$. The reaction mixture was cooled and poured gradually onto crushed ice. The resulting product was filtered, dried, and crystallized from ethanol.

7-Chloro-2-methylsulphanyl-5-phenylpyrazolo[1,5-a] pyrimidine3-carbonitrile (12): Yield: 73\%. M.p.: $196-197{ }^{\circ} \mathrm{C}$. FT-IR $\left(\mathrm{cm}^{-1}\right)$ $2220(\mathrm{CN}) .{ }^{1} \mathrm{H}$ NMR $\left(200 \mathrm{MHz}, \mathrm{DMSO}-d_{6}, \delta \mathrm{ppm}\right): 2.7\left(\mathrm{~s}, 3 \mathrm{H}, \mathrm{SC} H_{3}\right)$, 7.5-8.2 (m, 6H, Ar- $H+H 6)$. Anal. Calcd. for $\mathrm{C}_{14} \mathrm{H}_{9} \mathrm{ClN}{ }_{4} \mathrm{~S}: \mathrm{C}, 55.90 ; \mathrm{H}$, 3.01; N, 18.62. Found: C, 56.00; H, 3.23; N, 18.20\%.

5-Chloro-7-methyl-2-methylsulphanylpyrazolo[1,5-a] pyrimidine-3-carbonitrile (13): Yield: 71\%. M.p.: 220-221 ${ }^{\circ} \mathrm{C}$. FT-IR $\left(\mathrm{cm}^{-1}\right): 2223(\mathrm{CN}) .{ }^{1} \mathrm{H}$ NMR $\left(200 \mathrm{MHz}, \mathrm{DMSO}-d_{6}, \delta \mathrm{ppm}\right): 2.6(\mathrm{~s}, 3 \mathrm{H}$, $\left.\mathrm{CH}_{3}\right), 2.7$ (s, 3H, $\left.\mathrm{SCH}_{3}\right), 7.5(\mathrm{~s}, 1 \mathrm{H}, \mathrm{H6})$. Anal. Calcd. for $\mathrm{C}_{9} \mathrm{H}_{7} \mathrm{ClN}_{4} \mathrm{~S}: \mathrm{C}$, 45.28; H, 2.95; N, 23.47. Found: C, 45.65; H, 2.96; N, 23.45\%.

2.2.7. General procedure for the synthesis of 2-methylsulphanyl5-phenyl-7-(substituted amino)pyrazolo[1,5-a]pyrimidine-3carbonitriles 14a-c 
A mixture of 7-chloropyrazolo[1,5- $a$ ]pyrimidine derivative $\mathbf{1 2}$ $(0.60 \mathrm{~g}, 0.002 \mathrm{~mol})$, the appropriate primary amine $(0.002 \mathrm{~mol})$ and triethylamine $(0.50 \mathrm{~mL})$ in absolute ethanol $(20 \mathrm{~mL})$ was heated under reflux for $9 \mathrm{~h}$. The reaction mixture was concentrated under reduced pressure, and the solid formed upon cooling was filtered, dried and crystallized from the suitable solvent.

7-Cyclohexylamino-2-methylsulphanyl-5-phenylpyrazolo[1,5-a] pyrimidine-3-carbonitrile (14a): (Crystallized from ethanol). Yield: 60\%. M.p.: 194-195 ${ }^{\circ}$ C. FT-IR ( $\left.\mathrm{cm}^{-1}\right): 3383(\mathrm{NH}), 2213(\mathrm{CN}) .{ }^{1} \mathrm{H}$ NMR (200 MHz, DMSO- $d_{6}, \delta$ ppm): 1.2-1.9 (m, $11 \mathrm{H}$, aliphatic- $\left.H\right), 2.7(\mathrm{~s}$, $\left.3 \mathrm{H}, \mathrm{SCH}_{3}\right), 7.0(\mathrm{~s}, 1 \mathrm{H}, H 6), 7.5-8.2(\mathrm{~m}, 5 \mathrm{H}, \mathrm{Ar}-\mathrm{H}), 7.8\left(\mathrm{~s}, 1 \mathrm{H}, \mathrm{NH}, \mathrm{D}_{2} \mathrm{O}\right.$ exchangeable). Anal. Calcd. for $\mathrm{C}_{20} \mathrm{H}_{21} \mathrm{~N}_{5} \mathrm{~S}$ : C, 66.08; H, 5.82; N, 19.26. Found: C, $66.20 ; \mathrm{H}, 5.60 ; \mathrm{N}, 19.39 \%$.

7-Anilino-2-methylsulphanyl-5-phenylpyrazolo[1,5-a]pyrimidine3-carbonitrile (14b): (Crystallized from acetic acid). Yield: 44\%. M.p.: 254-255 ${ }^{\circ}$ C. FT-IR ( $\left.\mathrm{cm}^{-1}\right): 3349$ (NH), 2211 (CN). ${ }^{1} \mathrm{H}$ NMR (200 MHz, DMSO- $d_{6}, \delta$ ppm): 2.8 (s, 3H, SCH$\left.H_{3}\right), 6.7-8.3(\mathrm{~m}, 11 \mathrm{H}, \mathrm{Ar}-H$ ), 10.3 (s, $1 \mathrm{H}, \mathrm{NH}, \mathrm{D}_{2} \mathrm{O}$ exchangeable). Anal. Calcd. for $\mathrm{C}_{20} \mathrm{H}_{15} \mathrm{~N}_{5} \mathrm{~S}$ : C, 67.20; H, 4.22; N, 19.59. Found: C, 67.05; H, 4.12; N, 19.84\%.

7-(4-Methoxyanilino)-2-methylsulphanyl-5-phenylpyrazolo [1,5a]pyrimidine-3-carbonitrile (14c): (Crystallized from ethanol). Yield: 70\%. M.p.: 192-193 ${ }^{\circ} \mathrm{C}$. FT-IR ( $\left.\mathrm{cm}^{-1}\right): 3305(\mathrm{NH}), 2211(\mathrm{CN})$. ${ }^{1} \mathrm{H}$ NMR $\left(200 \mathrm{MHz}, \mathrm{DMSO}-d_{6}, \delta \mathrm{ppm}\right): 2.8\left(\mathrm{~s}, 3 \mathrm{H}, \mathrm{SCH}_{3}\right), 3.8(\mathrm{~s}, 3 \mathrm{H}$, $\mathrm{OCH}_{3}$ ), 6.5-7.9 (m, $\left.10 \mathrm{H}, \mathrm{Ar}-\mathrm{H}\right), 8.2\left(\mathrm{~s}, 1 \mathrm{H}, \mathrm{N} H, \mathrm{D}_{2} \mathrm{O}\right.$ exchangeable). Anal. Calcd. for $\mathrm{C}_{21} \mathrm{H}_{17} \mathrm{~N}_{5} \mathrm{OS}$ : C, 65.09; H, 4.42; N, 18.07. Found: C, $65.00 ; \mathrm{H}, 4.23 ; \mathrm{N}, 17.77 \%$.

\subsubsection{General procedure for the synthesis of 7-methyl-2- methylsulphanyl-5-(substituted amino)pyrazolo[1,5-a] pyrimidine-3-carbonitriles 15a-d}

A mixture of 5-chloropyrazolo[1,5-a]pyrimidine derivative 13 $(0.48 \mathrm{~g}, 0.002 \mathrm{~mol})$, the appropriate primary amine $(0.002 \mathrm{~mol})$ and triethylamine $(0.50 \mathrm{~mL})$ in absolute ethanol $(20 \mathrm{~mL})$ was heated under reflux for $11 \mathrm{~h}$. The reaction mixture was concentrated under reduced pressure, and the product formed upon cooling was filtered, dried and crystallized from ethanol.

5-Cyclohexylamino-7-methyl-2-methylsulphanylpyrazolo[1,5-a] pyrimidine-3-carbonitrile (15a): Yield: $91 \%$. M.p.: $180-181{ }^{\circ} \mathrm{C}$. FT-IR ( ${ }^{-1}$ ): $3378(\mathrm{NH}), 2211(\mathrm{CN}) .{ }^{1} \mathrm{H}$ NMR $\left(200 \mathrm{MHz}\right.$, DMSO- $\left.d_{6}, \delta \mathrm{ppm}\right):$ 1.2-1.9 (m, $11 \mathrm{H}$, aliphatic- $H$ ), $2.4\left(\mathrm{~s}, 3 \mathrm{H}, \mathrm{CH}_{3}\right), 2.7\left(\mathrm{~s}, 3 \mathrm{H}, \mathrm{SCH}_{3}\right), 6.4$ (s, 1H, H6), 7.7 (br s, $1 \mathrm{H}, \mathrm{N} H, \mathrm{D}_{2} \mathrm{O}$ exchangeable). Anal. Calcd. for $\mathrm{C}_{15} \mathrm{H}_{19} \mathrm{~N}_{5} \mathrm{~S}$ : C, 59.77; H, 6.35; N, 23.23. Found: C, 59.55; H, 6.31; N, $22.92 \%$.

5-Anilino-7-methyl-2-methylsulphanylpyrazolo[1,5-a]pyrimidine-3-carbonitrile (15b): Yield: 77\%. M.p.: 209-210 ${ }^{\circ} \mathrm{C}$. FT-IR ( $\mathrm{cm}^{-}$ 1): $3215(\mathrm{NH}), 2230(\mathrm{CN}) .{ }^{1} \mathrm{H}$ NMR $\left(200 \mathrm{MHz}\right.$, DMSO- $\left.d_{6}, \delta \mathrm{ppm}\right): 2.3$ (s, 3H, $\left.\mathrm{CH}_{3}\right), 2.7$ (s, 3H, $\left.\mathrm{SCH}_{3}\right), 6.2$ (s, $\left.1 \mathrm{H}, H 6\right), 7.1-7.5$ (m, $5 \mathrm{H}, \mathrm{Ar}-H$ ), $10.0\left(\mathrm{~s}, 1 \mathrm{H}, \mathrm{N} H, \mathrm{D}_{2} \mathrm{O}\right.$ exchangeable). Anal. Calcd/ for $\mathrm{C}_{15} \mathrm{H}_{13} \mathrm{~N}_{5} \mathrm{~S}$ : C, $60.99 ; \mathrm{H}, 4.43$; N, 23.71. Found: C, 60.86; H, 4.60; N, 23.45\%.

5-(4-Methoxyanilino)-7-methyl-2-methylsulphanylpyrazolo [1,5a]pyrimidine-3-carbonitrile (15c): Yield: 59\%. M.p.: 168-169 ㄷ. FTIR $\left(\mathrm{cm}^{-1}\right)$ : $3362(\mathrm{NH}), 2217(\mathrm{CN}) .{ }^{1} \mathrm{H}$ NMR $\left(200 \mathrm{MHz}\right.$, DMSO- $d_{6}, \delta$ ppm): $2.3\left(\mathrm{~s}, 3 \mathrm{H}, \mathrm{CH}_{3}\right), 2.7\left(\mathrm{~s}, 3 \mathrm{H}, \mathrm{SCH}_{3}\right), 3.8\left(\mathrm{~s}, 3 \mathrm{H}, \mathrm{OCH}_{3}\right), 6.0(\mathrm{~s}, 1 \mathrm{H}$, H6), 7.0 (d, $2 \mathrm{H}, J=8.7 \mathrm{~Hz}, \mathrm{Ar}-H$ ), 7.3 (d, $2 \mathrm{H}, J=8.7 \mathrm{~Hz}, \mathrm{Ar}-H$ ), 9.9 (s, $1 \mathrm{H}, \mathrm{NH}, \mathrm{D}_{2} \mathrm{O}$ exchangeable). MS (m/z (\%)): $325\left[\mathrm{M}^{+}, 100 \%\right]$. Anal. Calcd. for $\mathrm{C}_{16} \mathrm{H}_{15} \mathrm{~N}_{5} \mathrm{OS}$ : C, 59.06; H, 4.64; N, 21.52. Found: C, 59.39; H, 5.10; N, $21.11 \%$

5-(2-Methylanilino)-7-methyl-2-methylsulphanylpyrazolo[1,5-a] pyrimidine-3-carbonitrile (15d): Yield: 53\%. M.p.: 192-193 ${ }^{\circ} \mathrm{C}$. FT-IR $\left(\mathrm{cm}^{-1}\right): 3368(\mathrm{NH}), 2214(\mathrm{CN}) .{ }^{1} \mathrm{H}$ NMR $\left(200 \mathrm{MHz}\right.$, DMSO- $\left.d_{6}, \delta \mathrm{ppm}\right):$ $2.2\left(\mathrm{~s}, 3 \mathrm{H}, \mathrm{CH}_{3} \mathrm{C}_{6} \mathrm{H}_{4}\right), 2.3\left(\mathrm{~s}, 3 \mathrm{H}, \mathrm{CH}_{3}\right), 2.7\left(\mathrm{~s}, 3 \mathrm{H}, \mathrm{SCH} \mathrm{H}_{3}\right), 5.7$ (s, $1 \mathrm{H}$, H6), 7.3-7.4 (m, 4H, Ar- $H$ ), 9.9 (s, $1 \mathrm{H}, \mathrm{N} H, \mathrm{D}_{2} \mathrm{O}$ exchangeable). Anal. Calcd. for $\mathrm{C}_{16} \mathrm{H}_{15} \mathrm{~N}_{5} \mathrm{~S}$ : C, 62.11; H, 4.88; N, 22.63. Found: C, 62.40; H, $4.55 ; \mathrm{N}, 22.29 \%$.

\subsection{Biological testing}

\subsubsection{Materials and methods}

The human colon tumor cell line (HCT116) was obtained as a gift from National Cancer Institute NCI, Maryland MD, USA. All chemicals and solvents were purchased from Sigma-Aldrich.

\subsubsection{Measurement of potential cytotoxicity}

The cytotoxic activity of some of the newly synthesized compounds was tested in vitro on human colon tumor cell line (HCT116) using Sulforhodamine-B stain (SRB) assay according to the method of Skehan et al. [17]. Cells were plated in 96-multiwell plate $\left(10^{4}\right.$ cells/well) for $24 \mathrm{~h}$ before treatment with the tested compounds to allow attachment of the cells to the wall of the plate. The tested compounds were dissolved in DMSO and diluted with saline to the appropriate volume. Different concentrations of the tested compound $(0,1,2.5,5$ and $10 \mu \mathrm{g} / \mathrm{mL})$ were added to the cell monolayer. Triplicate wells prepared for each individual dose. Monolayer cells were incubated with the tested compound for $48 \mathrm{~h}$ at $37{ }^{\circ} \mathrm{C}$ in atmosphere of $5 \% \mathrm{CO}_{2}$. After $48 \mathrm{~h}$, cells were fixed with trichloroacetic acid, washed with water and stained for $30 \mathrm{~min}$ with $0.4 \%$ (w:v) Sulforhodamine-B stain dissolved with 1\% acetic acid. Excess stain was removed by four washes with $1 \%$ acetic acid and attached stain was recovered with Tris EDTA buffer. Colour intensity was measured in ELISA reader. The relation between surviving fraction and compound concentration was plotted and $\mathrm{IC}_{50}$ (the concentration required for $50 \%$ inhibition of cell viability) was calculated for each compound and results are given in Table 1. Figure 2 represents $\mathrm{IC}_{50}$ in $\mu \mathrm{M}$ of the synthesized compounds and doxorubicin against human colon tumor cell line (HCT116)

Table 1. Results of in vitro cytotoxic activity of some of the synthesized compounds on human colon tumor cell line (HCT116).

\begin{tabular}{cc}
\hline compounds on human colon tumor cell line (HCT116). \\
\hline Compound no. & IC $_{\mathbf{5 0}}$ in $\boldsymbol{\mu M}^{*}$ \\
\hline Doxorubicin & 0.0069 \\
$7 \mathrm{~b}$ & 0.0062 \\
$7 \mathrm{c}$ & 0.0375 \\
8 & 0.0433 \\
9 & 0.0814 \\
$14 \mathrm{a}$ & 0.0020 \\
$14 \mathrm{~b}$ & 0.0113 \\
$14 \mathrm{c}$ & 0.0238 \\
$15 \mathrm{c}$ & 0.0077 \\
$15 \mathrm{~d}$ & 0.0598 \\
\hline
\end{tabular}

*The values given are means of three experiments.

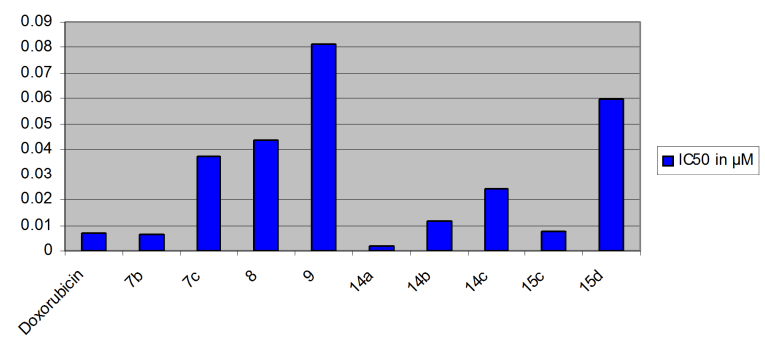

Figure 2. $\mathrm{IC}_{50}$ in $\mu \mathrm{M}$ of the synthesized compounds and doxorubicin against human colon tumor cell line (HCT116).

\section{Results and discussion}

\subsection{Chemistry}

The synthesis of the target compounds is outlined in Schemes 1 and 2. The starting compound, 5-amino-3-methylsulphanyl-1Hpyrazole-4-carbonitrile (4) [11] was reacted with the appropriate substituted benzylidenemalononitrile 5a-f [12-15] to afford 7amino-2-methylsulphanyl-5-(substituted phenyl)-4,5-dihydropyrazolo[1,5-a]pyrimidine-3,6-dicarbonitriles $\mathbf{6 a}, \mathbf{b}$ or 7-amino-2methylsulphanyl-5-(substituted phenyl)pyrazolo[1,5- $a$ ]pyrimidine3,6-dicarbonitriles 7a-d.

The reaction of the pyrazole derivative $\mathbf{4}$ with substituted benzylidenemalononitriles 5a-f may proceed via initial nucleophilic attack by the exocyclic amino group of compound 4 on the activated double bond in $\mathbf{5 a - f}$ to form a Micheal adduct Intramolecular cyclization of the latter may result in the formation of the dihydro derivative $\mathbf{6}$ which may then aromatize into 7 (Scheme 3). 


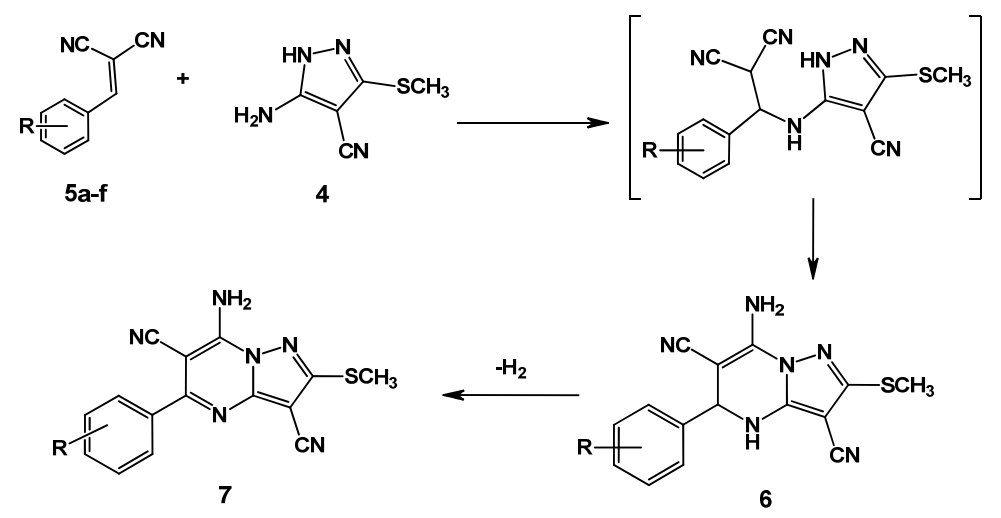

Scheme 3

Trials to oxidize $\mathbf{6 a}, \mathbf{b}$ into $7\left(\mathrm{R}=2-\mathrm{Cl}, 2-\mathrm{CH}_{3} \mathrm{O}\right)$ via the use of two molar equivalents of benzylidenemalononitriles $\mathbf{5 a}, \mathbf{b}$ or by increasing the time of the reaction (up to $15 \mathrm{~h}$ ) were unsuccessful.

Compound 4 was also reacted with malononitrile to give 5,7diaminopyrazolo[1,5-a]pyrimidine 8. ${ }^{1} \mathrm{H}$ NMR spectrum of compound 8 revealed the presence of a singlet signal at $\delta 5.3 \mathrm{ppm}$ corresponding to $\mathrm{H} 6$ and two exchangeable singlet signals at $\delta 6.7$ and $\delta 7.3 \mathrm{ppm}$ corresponding to protons of the two $\mathrm{NH}_{2}$ groups at positions 5 and 7 , respectively.

Furthermore, $1 H$-pyrazole derivative 4 was reacted with ethyl cyanoacetate, ethyl benzoylacetate and ethyl acetoacetate to give one of two possible products; the 5-oxopyrazolo[1,5-a]pyrimidine or the 7-oxo isomer (Scheme 4). The assignment of the product as 5-oxo or 7-oxopyrazolo[1,5-a]pyrimidine depends on ${ }^{1} \mathrm{H}$ NMR study.<smiles>[R]c1cc(=O)[nH]c2c(C#N)c(C)nn12</smiles>

5-Oxopyrazolo[1,5-a]pyrimidine

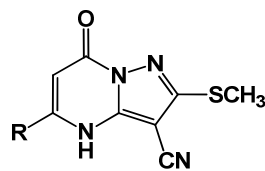

7-Oxopyrazolo[1,5-a]pyrimidine

$\mathrm{R}=\mathrm{NH}_{2}, \mathrm{C}_{6} \mathrm{H}_{5}, \mathrm{CH}_{3}$

Scheme 4

The reaction of compound $\mathbf{4}$ with ethyl cyanoacetate and ethyl acetoacetate afforded 5-oxopyrazolo[1,5- $a$ ]pyrimidine derivatives 9 and 11, respectively. Both compounds showed an exchangeable singlet signal at $\delta 11.9 \mathrm{ppm}$ and $\delta 13.2 \mathrm{ppm}$, respectively, corresponding to $\mathrm{NH}$ proton due to tautomerism with the adjacent carbonyl group. The 7-oxo isomer, lacking such tautomerism, should give that signal at $\delta 5-7 \mathrm{ppm}$.

On the other hand, the reaction of compound 4 with ethyl benzoylacetate afforded 7-oxopyrazolo[1,5-a]pyrimidine $\mathbf{1 0}$. Herein, ${ }^{1} \mathrm{H}$ NMR of compound $\mathbf{1 0}$ revealed the presence of an exchangeable singlet signal at $\delta 6.2$ ppm corresponding to $\mathrm{NH}$ proton.

Chlorination of compounds $\mathbf{1 0}$ and $\mathbf{1 1}$ with $\mathrm{POCl}_{3}$ followed by nucleophilic substitution with primary amines afforded 7(substituted amino) and 5-(substituted amino)pyrazolo[1,5a]pyrimidine derivatives $\mathbf{1 4 a - c}$ and $\mathbf{1 5 a}-\mathbf{d}$, respectively.

\subsection{In vitro anticancer screening}

Some of the newly synthesized compounds were evaluated for their in vitro cytotoxic activity against human colon tumor cell line, HCT116. Doxorubicin which is one of the most effective anticancer agents was used as the reference drug in this study.

The response parameter calculated was the $\mathrm{IC}_{50}$ value, which corresponds to the concentration required for 50\% inhibition of cell viability. The $\mathrm{IC}_{50}$ in $\mu \mathrm{M}$ of the tested compounds compared to the reference drug are shown in Table 1 and represented graphically in Figure 2.

From the results in Table 1, it was found that all the tested compounds exhibited good antitumor activity against HCT116 with IC 50 between 0.0020 and $0.0814 \mu \mathrm{M}$.

Two of the tested compounds (7b and 14a) showed antitumor activity superior to doxorubicin with $\mathrm{IC}_{50}$ that equals to 0.0069 and $0.0020 \mu \mathrm{M}$, respectively.

The best results were obtained by 5 -phenyl-7-(substituted amino)pyrazolo[1,5-a]pyrimidine derivatives 14a-c, and the highest activity was obtained by 7-cyclohexylamino-2methylsulphanyl-5-phenylpyrazolo[1,5-a]pyrimidine-3carbonitrile (14a)

\section{Conclusion}

In summary, a novel series of pyrazolo[1,5- $a$ ]pyrimidine-3carbonitriles substituted with 7-amino (6-9), 7-substituted amino (14a-c) and 5-substituted amino (15a-d) was synthesized. Some of the newly synthesized compounds were tested in vitro on human colon tumor cell line (HCT116). Most of the tested compounds exhibited good antitumor activity, especially compound 14a which displayed the highest activity among the tested compounds with $\mathrm{IC}_{50}$ that equals to $0.0020 \mu \mathrm{M}$.

\section{Acknowledgements}

We are grateful to Prof. Dr. Samia Shouman, Professor of Pharmacology, and all members of the department of Cancer Biology, National Cancer Institute, Cairo, Egypt, for carrying out the cytotoxicity testing.

\section{References}

[1]. Waller, D. G.; Renwick, A. G.; Hillier, K.; Medical Pharmacology and Therapeutics, 2nd Edn., 2005, pp. 635.

[2]. Fraley, M. E.; Rubino, R. S.; Hoffman, W. F.; Hambaugh, S. R. Bioorg Med. Chem. Lett. 2002, 12, 3537-3541.

[3]. Williamson, D. S.; Parratt, M. J.; Bower, J. F.; Moore, J. D.; Richardson, C M.; Dokurno, P.; Cansfield, A. D.; Francis, G. L.; Hebdon, R. J.; Howes, R.; Jackson, P. S.; Lockie, A. M.; Murray, J. B.; Nunns, C. L.; Powles, J.; Robertson, A.; Surgenor, A. E.; Torrance, C. J. Bioorg. Med. Chem. Lett. 2005, 15, 863-867.

[4]. Gopalsamy, A.; Yang, H.; Ellingboe, J. W.; Tsou, H.; Zhang, N.; Honores, E.; Powell, D.; Miranda, M.; McGinnis, J. P.; Rabindran, S. K. Bioorg. Med. Chem. Lett. 2005, 15, 1591-1594.

[5]. Li, J.; Zhao, Y. F.; Zhao, X. L.; Yuan, X. Y.; Gong, P. Arch. Pharm. Chem Life Sci. 2006, 339, 593-597

[6]. Guzi, T. J.; Paruch, K.; Dwyer, M. P.; (Schering Corporation, USA) U.S Pat. Appl. Publ. US 2006 41,131 (Cl. 544-281; A61K31/519), 23 Feb 
2006, US Appl. 2003/653,776, 3 Sep 2003; 85 pp. (Eng), Through Chem. Abstr. 2006, 144, 254145y.

[7]. Paruch, K.; Dwyer, M. P.; Alvarez, C.; Brown, C.; Chan, T.; Doll, R. J.; Keertikar, K.; Knutson, C.; McKittrick, B.; Rivera, J.; Rossman, R.; Tucker, G.; Fischmann, T. 0.; Hruza, A.; Madison, V.; Nomeir, A. A.; Wang, Y.; Lees, E.; Parry, D.; Sgambellone, N.; Seghezzi, W.; Schultz, L.; Shanahan, F.; Wiswell, D.; Xu, X.; Zhou, Q.; James, R. A.; Paradkar, V. M.; Park, H.; Rokosz, L. R.; Stauffer, T. M.; Guzi, T. J. Bioorg. Med. Chem. Lett. 2007, 17, 6220-6223.

[8]. Dwyer, M. P.; Paruch, K.; Alvarez, C.; Doll, R. J.; Keertikar, K.; Duca, J.; Fischmann, T. O.; Hruza, A.; Madison, V.; Lees, E.; Parry, D.; Seghezzi, W.; Sgambellone, N.; Shanahan, F.; Wiswell, D.; Guzi, T. J. Bioorg. Med. Chem. Lett. 2007, 17, 6216-6219.

[9]. Powell, D.; Gopalsamy, A.; Wang, Y. D.; Zhang, N.; Miranda, M.; McGinnis, J. P.; Rabindran, S. K. Bioorg. Med. Chem. Lett. 2007, 17, 1641-1645.

[10]. Wang, Y. D.; Honores, E.; Wu, B.; Johnson, S.; Powell, D.; Miranda, M.; McGinnis, J. P.; Discafani, C.; Rabindran, K.; Cheng, W.; Krishnamurthy, G. Bioorg. Med. Chem. 2009, 17, 2091-2100.

[11]. Gompper, R.; Toepfl, W. Chem. Ber. 1962, 95, 2881-2884.

[12]. Corson, B. B.; Stoughton, R. W. J. Am. Chem. Soc. 1928, 50, 2825-2831.

[13]. Baker, W.; Howes, C. S. J. Chem. Soc. 1953, 119-124.

[14]. Patai, S.; Israeli, Y. J. Chem. Soc. 1960, 2020-2024

[15]. Zabicky, J. Chem. Soc. 1961, 683-686.

[16]. Sadek, K. U.; Selim, M. A.; Elnagdi, M. H.; Otto, H. H. Bull. Chem. Soc. Jpn. 1993, 66, 2927-2930.

[17]. Skehan, P.; Storeng, R.; Scudiero, D.; Monks, A.; McMahon, J.; Vistica, D.; Warren, J. T.; Bokesch, H.; Kenney, S.; Boyd, M. R. J. Natl. Cancer Inst. 1990, 82, 1107-1112. 